\title{
Database screening of herbal monomers regulating autophagy by constructing a "disease-gene-drug" network
}

Chenjun Hao ${ }^{1 \dagger}$, Zhengpeng Yang ${ }^{1 \dagger}$, Bo Gao ${ }^{1}$, Ming Lu², Xianzhi Meng ${ }^{1}$, Xin Qiao ${ }^{2}$, Dongbo Xue ${ }^{1 *}$ and Weihui Zhang ${ }^{1 *}$

\begin{abstract}
Background: Studies suggest an important role of autophagy as a target for cancer therapy. We constructed a "disease-gene-drug" network using the modular approach of bioinformatics and screened herbal monomers demonstrating functions related to autophagy regulation.

Methods: Based on the microarray results of the gene expression omnibus (GEO) database (GSE2435 and GSE31040, starvation-induced autophagy model), we used the human protein reference database (HPRD) to obtain the protein-protein interaction (PPI) network. In addition, we used the CFinder software to identify several functional modules, performed gene ontology-biological process (GO-BP) functional enrichment analysis using the DAVID software, constructed a herbal monomer-module gene regulatory network using literature search and the Cytoscape software, and then analyzed the relationships between autophagy, genes, and herbal monomers.

Results: We screened 544 differentially expressed genes related to autophagy, 375 pairs of differentially expressed genes, and 7 gene modules, wherein the functions of module 3 (composed of 7 genes) were enriched in "cell death". Using the constructed herbal monomer-module gene regulatory network, we found that 30 herbal monomers can simultaneously regulate these 7 genes, indicating a potential regulatory role in autophagy.

Conclusions: Database screening using the disease-gene-drug network can provide new strategies and ideas for the application of herbal medicines in cancer therapy.
\end{abstract}

Keywords: Herbal monomer, Autophagy, Genomics, Bioinformatics

\section{Background}

For several thousands of years, natural products have been used as medicines. Indeed, since ancient times, humans have searched for naturally occurring substances to alleviate their sufferings. Before the invention of synthetic drugs, natural drugs were the only choice for treating diseases. Even today, many of the world's populations still use natural medicine to treat diseases, including traditional Chinese medicine. With the development of science and technology, in-depth research has been conducted on ancient drugs using modern approaches

\footnotetext{
*Correspondence: Dongbo_xue@126.com; zhangweihui626@hotmail.com ${ }^{\dagger}$ Equal contributors

'Department of General Surgery, the First Affiliated Hospital of Harbin Medical University, Harbin, China

Full list of author information is available at the end of the article
}

to isolate effective monomers from complex natural mixtures, and remarkable achievements have been made. Natural products are important sources of new anti-cancer drugs, as more than $60 \%$ of anti-cancer drugs have been derived from natural products. Recently, a significant number of anti-cancer drugs derived from natural products, especially drugs of plant origins such as rhoeadine, podophyllotoxin, paclitaxel and camptothecin, have achieved great success in clinical application [1,2].

Autophagy is an evolutionarily conserved process involved in homeostasis by degrading organelles and proteins to maintain biosynthesis during nutrient deprivation or metabolic stress [3]. This process is important for the elimination of damaged organelles and proteins with long half-lives. Defect in autophagy is associated 
with tumorigenesis via metabolic stress and DNA damage [4]. Loss of Beclin-1, an essential autophagy gene, is present in $40-75 \%$ of solid tumors, suggesting a tumor suppresion role of autophagy [4]. Although autophagy has been identified as a type of programmed cell death [5-7], autophagy is also a pro-survival process that enables tumor cells to survive adverse conditions such as hypoxia and chemotherapy [3,8]. Autophagy is a pathway that is distinct from apoptosis, although some crosstalk exists between them $[9,10]$. Therefore, because autophagy plays a complex and very important role in the pathogenesis and development of tumors, this process has become an important anti-cancer therapy target. As a result, approaches to effectively regulate autophagy are increasingly becoming the subject of active research.

In order to systematically identify herbal monomers for potential application in tumor treatment with regards to their ability to regulate autophagy, this study used the approaches of modular bioinformatics analysis and network pharmacology. Theoretically, if the inhibition of a certain gene can affect the pathogenesis and development of a disease, drugs regulating this gene might be effective for its treatment. In addition, it has been noted that disease occurrence is the result of changes in the transcription of multiple genes or gene groups in the human genome. Therefore, drug treatment should also target the transcription of these genes or gene groups in order to be more effective. Integrated analysis using "disease-gene" and "gene-drug" networks facilitate the identification of herbal monomers regulating autophagy, providing novel uses for old drugs.

\section{Methods}

\section{Microarray data processing}

Two series of Earle's balanced salt solution (EBSS) starvation-induced autophagy model in a human lymphoblastoid cell line were selected in this study, including GSE2435 (http://www.ncbi.nlm.nih.gov/geo/query/acc.cgi? acc=GSE2345) [11] and GSE31040 (http://www.ncbi.nlm. nih.gov/geo/query/acc.cgi?acc) [12], which can be obtained from the GEO (Gene Expression Omnibus) database. The differentially expressed genes were detected based on the HG-U133-Plus-2 Affymetrix Human Genome U133 Plus 2.0 Array. Each experiment was repeated three times.

The microarray experiments performed in this study included the controls and the autophagy conditions in human cells, with three samples for each condition. We downloaded the microarray CEL data compression package from the GEO supplementary file and extracted the package to a folder for later use. We also downloaded the raw data in TXT format. We used Bioconductor and $\mathrm{R} 2.10 .1$ to normalize the downloaded data. A robust multi-array average (RMA) algorithm was applied to calculate the expression levels, and a multiscale adaptive search (MAS) algorithm was used to calculate the detection calls. Each group of samples with no less than 2 detection calls was retained and called Present (P), in order to filter low-expression probe sets. The HUGO Gene Nomenclature Committee (HGNC) gene names for human genes were used to unify the gene names. We then used the VLOOKUP function to replace the probe numbers in the original data and established the expression data sheet containing the gene names and the corresponding samples. We used the LIMMA differentially expressed gene screen algorithm to screen genes either upregulated or downregulated in the autophagy condition compared with the control condition, and the fold of change, P-value and false discovery rate (FDR) were calculated. P-values $<0.5$ or $>2$ indicated differentially expressed genes.

The intersection between GSE2435 and GSE31040 was used to screen the differentially expressed genes shared by the two microarrays.

\section{Generation of differentially expressed gene modules and functional analysis of the modules}

We obtained the protein-protein interaction (PPI) network from the Human Protein Reference Database (HPRD). This network contains 36,874 edges and 9,453 nodes [13]. The differentially expressed genes were mapped to the PPI network, and pairs in which the two interacting proteins were both differentially expressed were retained. Subsequently, sub-networks were generated.

We used the CFinder software (http://cfinder.org/) to identify functional modules in the sub-networks. CFinder [14] was used for finding and visualizing concealed groups and modules in networks, based on the clique percolation method (CPM). CFinder can find the full join set of a specified size in the network and construct larger node groups through the shared nodes and edges in the full join set.

We used the Database for Annotation, Visualization and Integrated Discovery (DAVID) software (v6.7) for gene ontology-biological process (GO-BP) analysis of the screened modules. We opened the DAVID database (http://david.abcc.ncifcrf.gov/), submitted the gene groups proposed for further analysis, checked the entire human genome as the background genes, selected "Functional Annotation Tool" as the analytical tool, and then obtained the GO-BP enrichment analysis results ( $\mathrm{P}$-value was 0.05).

A file of the interesting genes and their corresponding GO-BP terms was generated. The file was imported to Cytoscape (version 2.6.3) [15], the interaction type was "default interaction", and the "layout", "cytoscape layout" and "spring embedded" options were selected. At last, a 
network illustrating the functions of interesting genes was constructed.

\section{Construction of the herbal monomer-module gene regulatory networks}

Based on the herbal monomer database (http://www.sepu. net/html/article), we established a file of herbal monomers including 30 monomers mightly related to autophagy, entered the file of differentially expressed module genes and the file of herbal monomers into the ActivePerl 5.16.2 software, and extracted a literature set from the PUBMED database that was related to "each differentially expressed module gene" and "each herbal monomer". We also conducted preliminary grammar and syntax analyses of the target literature set, analyzed the potential conceptual relationship in the literature, and identified the corresponding herbal monomers regulating target genes, followed by manual screening. We then drew the herbal monomermodule gene regulatory network diagram using the Cytoscape software (version 2.6.3).

\section{Results}

Detection of differentially expressed genes after autophagy induction

GSE2435 detected a total of 13,234 genes. Taking a P-value $<0.05$ as the significance level of differentially expressed genes, we screened a total of 2783 differentially expressed genes. GSE31040 detected a total of 14,442. Taking a P-value $<0.05$ as the significance level for differentially expressed genes, we screened a total of 2,369 differentially expressed genes. There were a total of 544 differentially expressed genes identified for the intersection of GSE2435 and GSE31040.

\section{Modular analysis results of autophagy-related genes}

According to the HPRD database, there were a total of 36,875 PPI pairs. The differentially expressed genes were then projected to the PPI pairs, and we ensured that only the pairs in which the two interacting proteins were both differentially expressed were retained. Eventually, we obtained 375 differentially expressed relationship pairs. We used the Cytoscape software to build the subnetwork diagram, and the results are shown in Figure 1.

The present study used the CFinder software to conduct module identification for the differentially expressed PPI pairs obtained from the steps described above. When selecting $\mathrm{k}=3$, a total of 7 modules was obtained, as shown in Figure 2.

We used the DAVID software to perform GO-BP functional enrichment analysis for each of the 7 modules. The functional analysis results showed that the functions enriched in module 3 were primarily related to "cell death" (Figure 3). We thus chose module 3 as the module of interest for further analysis.
There seven genes within module 3, including NFKBIA, GADD45A, GADD45B, CDKN1A, JUNB, PPP1R15A and $A T F 3$. We constructed the relationship diagram for each gene and the corresponding GO-BP term using the Cytoscape software (Figure 4). The results indicated that GADD45A, GADD45B, PPP1R15A and NFKBIA were strongly related to "cell death".

\section{Construction and analysis of gene-drug networks}

The gene-drug network diagram of the seven genes in module 3 and the herbal monomer library were constructed by literature mining (Figure 5). These results showed that the effects of some herbal monomers were multitargeted. Among them, 30 herbal monomers in group E can act simultaneously on the 7 genes in module 3, which were selected as the focus of our study.

\section{Discussion}

Programmed cell death is a spontaneous cell death process committed to by cells with damage that cannot be repaired, in order to maintain homeostasis. There are three main types of programmed cell death: apoptosis, autophagy and necrosis [3]. Autophagy itself is a mechanism by which abnormal intracellular components are degraded to achieve homeostasis [3]. However, while presenting increased autophagic activity, cell death induced by many types of stress also shows morphological characteristics that do not fully comply with apoptosis or necrosis. Therefore, autophagy is considered a distinct mechanism of cell death $[9,10]$. Autophagy plays an extremely important and complicated role in the pathogenesis and development of tumors. Studies have confirmed that autophagy is lower in tumor cells compared with normal cells in the same tissue during the early phase of tumorigenesis. Some tumors, such as liver cancer caused by chemical carcinogens, already demonstrate reduced autophagic capacity during the early phase of tumorigenesis. However, some other cancers (such as pancreatic cancer) showed increased autophagy during tumorigenesis [16]. After tumor induction by carcinogens, rat pancreas cells demonstrated normal autophagic capacity in precancerous nodules and adenomas, whereas this level of autophagy was reduced upon the formation of cancer cells [17]. Autophagy can first remove organelles (primarily mitochondria) damaged by chemical carcinogens, radiation and oxidative stress, thus avoiding genetic instability due to DNA damage caused by reactive oxygen species (ROS) and reducing the incidence of tumors. In addition, autophagy can degrade the endoplasmic reticulum, Golgi apparatus and other organelles, as well as proteins with long half-lives. Therefore, pre-malignant cells are in a negative balance of protein, which inhibits uncontrolled proliferation [18]. Indeed, excessive autophagic cell death can kill tumor cells, indicating the anti- 

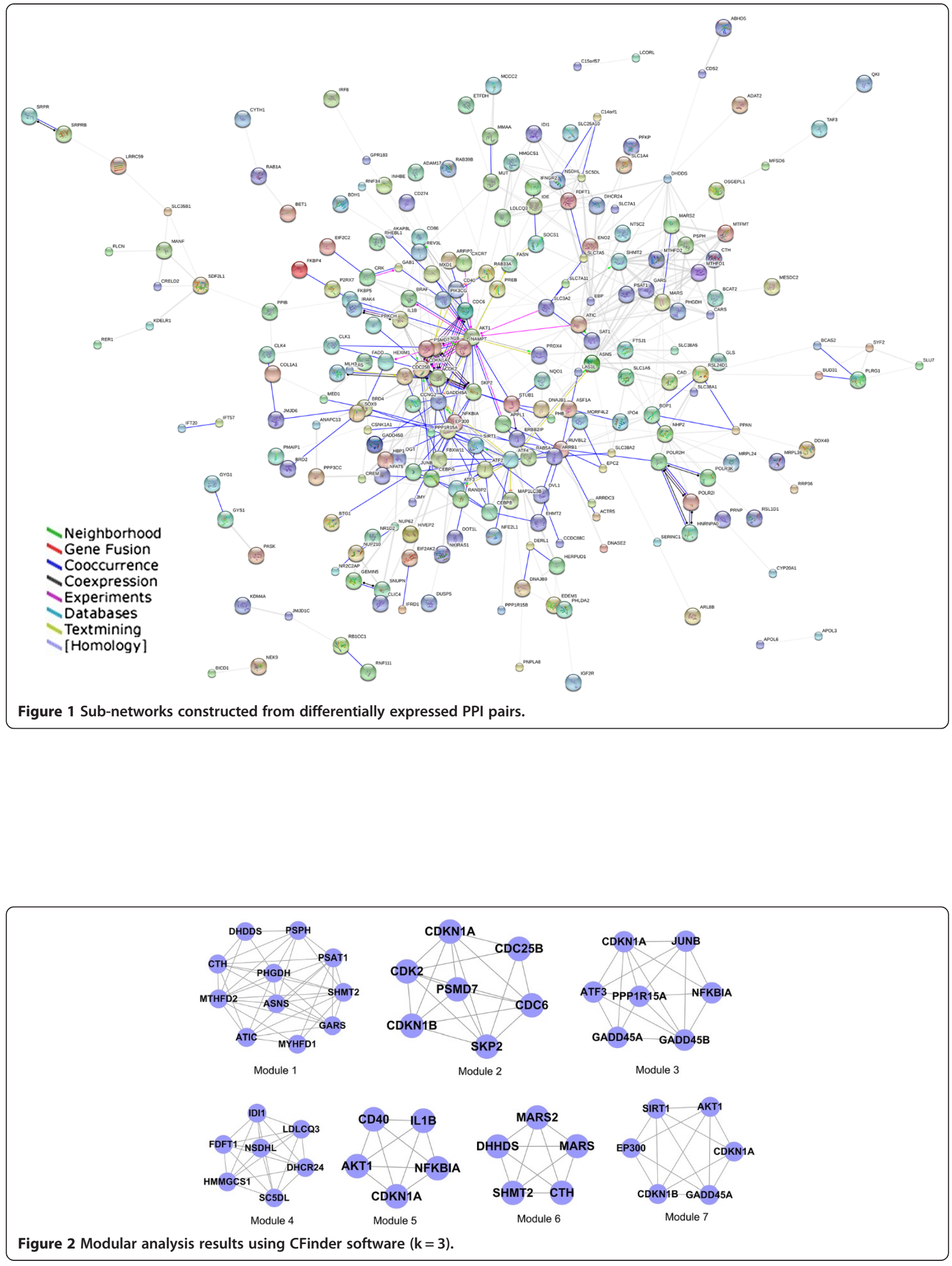


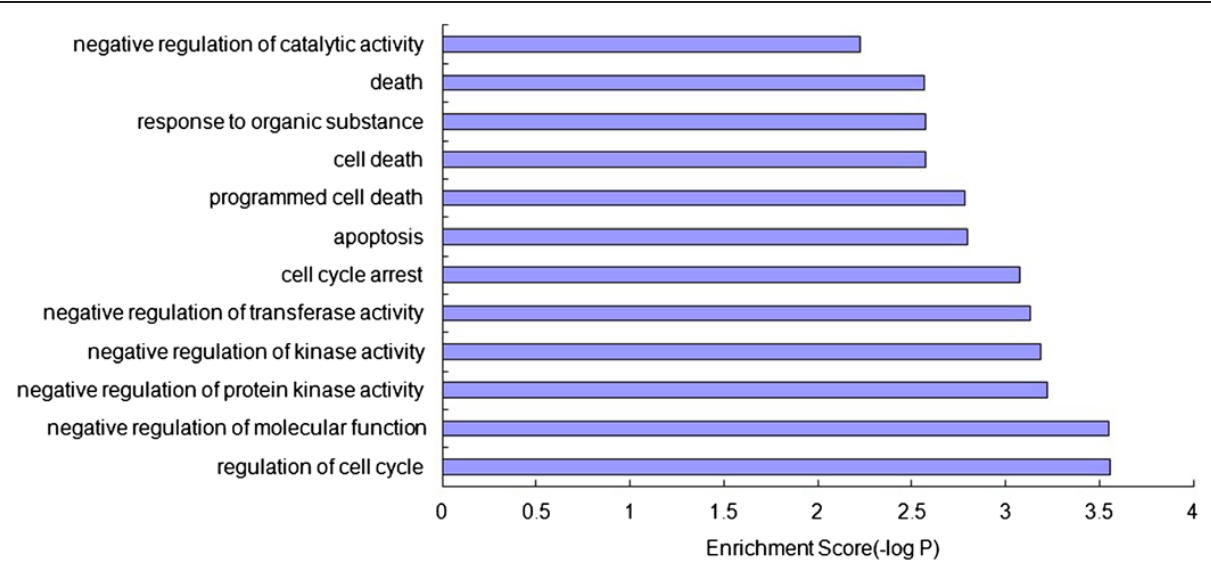

Figure 3 GO-BP functional enrichment analyses of genes in module 3.

cancer effect of autophagy. For example, tamoxifen can induce autophagic cell death in MCF-7 cells [19], and arsenic trioxide can stimulate autophagy in lymphocytic leukaemia and multiple myeloma cells [20]. After irradiation, $\mathrm{Bax}^{-1-} \mathrm{Bak}^{-/-}$mouse embryonic fibroblast (MEF) cells showed reduced Akt/mTOR signalling and increased expression of ATG5, ATG12, Beclin1 and other autophagic factors; moreover, after Rad001 (mTOR inhibitor) treatment, autophagy was further enhanced, and MEF cells presented autophagic cell death [21]. Therefore, studies focusing on the regulatory mechanisms of autophagy and effective interventions for regulating autophagy have become an active area of cancer treatment research.

In this study, we focused on autophagy regulation and used microarray results from the GEO database to perform modular bioinformatics analysis using the CFinder, DAVID and Cytoscape software. We screened a gene group (module 3) composed of seven closely linked genes among the differentially expressed genes after the induction of autophagy. The functions of this gene group were primarily enriched in "cell death". In particular, four genes, GADD45A, GADD45B, PPP1R15A and NFKBIA, were directly related to "cell death".

NFKBIA ( $\mathrm{IkB} \alpha)$ encodes an inhibitor of NF-kB that regulates the activity of NF- $\mathrm{KB}$ by binding to and dissociating from NF-kB [22]. Studies have shown that in acute pancreatitis [23], the NF- $\mathrm{BB}$ signaling pathway regulates the expression of microtubule-associated protein light chain 3 (LC3) and Beclin-l to regulate the occurrence of pancreatic autophagy in rat acute pancreatitis. Genetic changes in NFKBIA are involved in colorectal [24] and glioblastoma tumorigenesis [25].

The GADD45 family consists of three genes (GADD45A, GADD45B and GADD45G) and have been shown to be involved in tumorigenesis [26]. The common feature of the GADD45 family is that the gene products can bind to the N-terminal domain of MTK1 and activate the MTK1 kinase and p38/JNK both in vivo and in vitro. It has also been shown that $\mathrm{p} 38$ plays a decisive role in the regulation of autophagy, and that SB203580 (a p38 specific inhibitor)

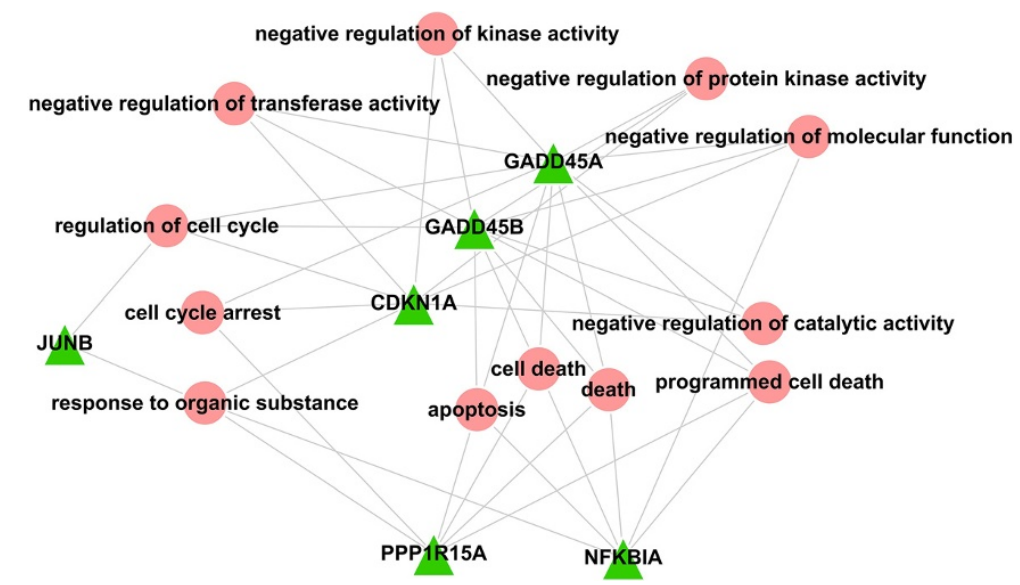

Figure 4 Network diagrams of genes in module 3 and the corresponding GO-BP term. Ellipse shape nodes represent GO-BP terms, and triangle shape nodes indicate genes. 


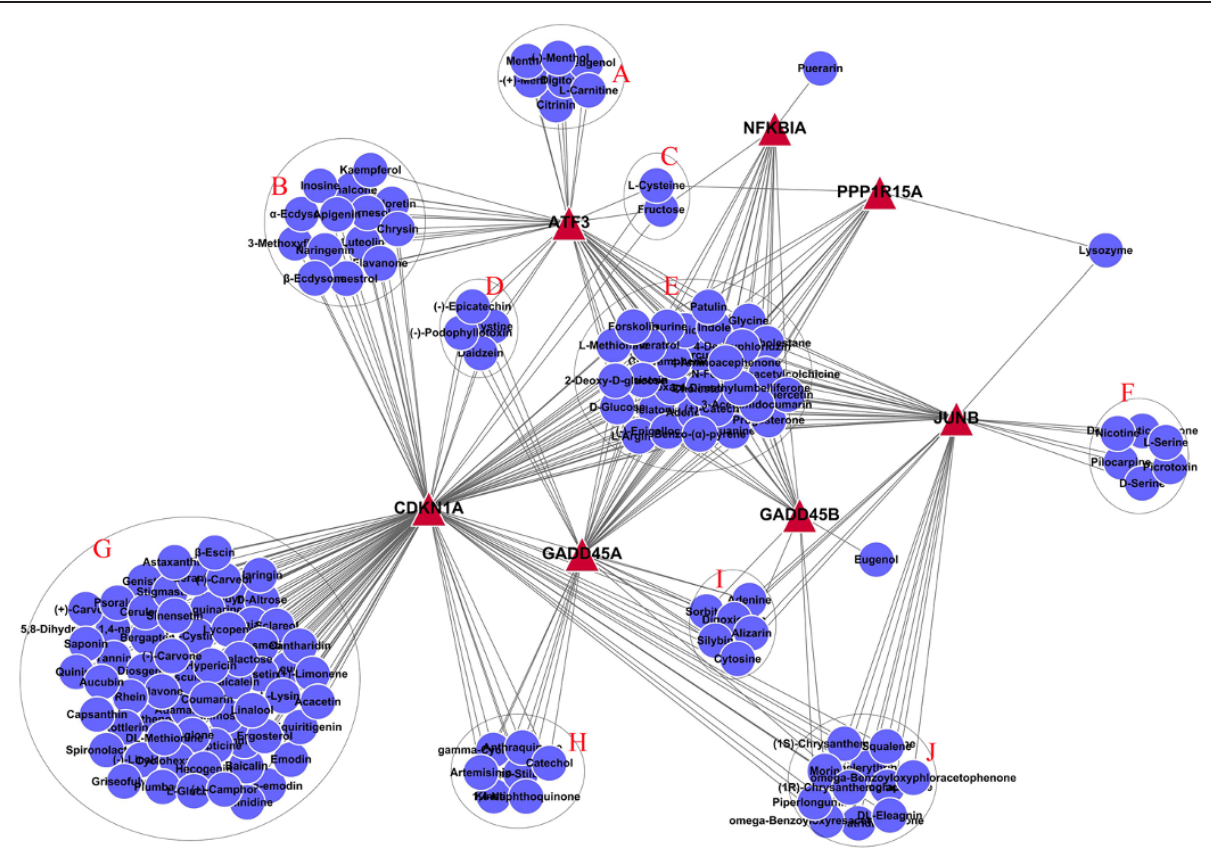

Figure 5 Network diagrams of the relationships between herbal monomers and genes in module 3. Ellipse shape nodes indicate herbal monomers, and triangle shape nodes indicate genes.

can block the autophagy process [27]. Recent studies have shown that JNK participates in CH11 (Fas agonistic antibody)-induced autophagy, and further experiments have shown that the JNK inhibitor SP600125 can hinder the process of autophagy [28], suggesting that JNK and p38 are closely related to cellular autophagy. It can therefore be inferred that the GADD45 family of genes are closely associated with autophagy.

$C D K N 1 A$, also known as p21, participates in cell cycle regulation and inhibits or mediates apoptosis-related signaling pathways [29]. It has also been reported that in ceramide-treated MEFs, interference of p21 expression in p $21^{+/+}$MEFs led to a decreased level of apoptosis and increased autophagy, whereas p21 overexpression in $\mathrm{p} 21^{-/-}$ MEFs enhanced autophagy and decreased the level of apoptosis [30]. CDKN1A has been shown to be associated with colorectal [31] and prostate cancer risk [32].

JUNB is also called Activator Protein 1 (AP-1) and is involved in skin [33] and breast cancer development [34]. Recent studies have found that in the process of autophagy, LC3 may bind specifically to the membrane structure of trans-Golgi network (TGN), and then TGN generates LC3-positive vesicles that bring LC3 to early autophagosome structures formed by the endoplasmic reticulum or other membrane structures [35]. In this process, the formation of AP-1 was shown to regulate clathrin vesicles together with ATG9 to regulate autophagy through the binding of LC3-PE to the corresponding complex and the formation of LC3-positive vesicles.
PPP1R15A (GADD34) dephosphorylates the eIF2 $\alpha$ protein [36]. In MEFs, herpes simplex virus-induced PKRdependent eIF $2 \alpha$ phosphorylation was shown to induce autophagy. It has also been reported that eIF $2 \alpha$ phosphorylation is necessary for amino acid deficiency-induced autophagy $[37,38]$. Thus, eIF2 $\alpha$ phosphorylation plays an important role in ER stress-induced autophagy.

From the above evidence, we can conclude that most of the genes in module 3 are closely associated with autophagy and that some of them are associated with cancer development. Because disease regulation by a single gene is not efficient, we introduced the concept of network pharmacology to examine how these genes are regulated, established a gene-drug network based on previously established disease-gene networks, and effectively combined these approaches to derive drugs for the treatment of diseases. The present study used the herbal monomer database to screen for monomers targeting the 7 genes in module 3. In particular, we performed literature mining of the PUBMED database and constructed the herbal monomer-module gene regulatory network using the Cytoscape software. We then selected 30 herbal monomers that could simultaneously act on these 7 genes and performed further literature searches. We found that herbal monomers such as resveratrol, curcumin, quercetin, and melatonin showed important relationships with autophagy.

Resveratrol is a polyphenol compound extracted from plants of the genus Vitis. Resveratrol possesses various biological functions, such as the induction of cell cycle 
arrest and apoptosis, as well as anti-tumor roles in vivo $[39,40]$. Yamamoto et al. [41] showed that resveratrol could induce autophagy in glial cells; in addition, P38, MAPK and ERK1/2 MAPK participate in resveratrolinduced glial cell autophagy. Choi et al. [42] found that resveratrol treatment could induce protective autophagy in human dermal fibroblasts (HDFs) cultured under normal conditions. It has also been reported in the literature [43] that resveratrol can induce autophagy in different tumor cell lines including ovarian cancer $[44,45]$, colorectal cancer [46], gastric cancer [47], lung cancer and salivary gland cancer $[48,49]$. Many pathways and mechanisms are involved in resveratrol-induced autophagy such as inhibition of the mTOR pathway and activation of the MAPK pathway $[44,50]$. In addition, resveratrol-induced autophagy stimulates an accumulation of PELP-1 (proline-, glutamic acid-, and leucine-rich protein-1), which is a class of estrogen receptor co-activator in autophagosomes [49], as well as activation of Vps34 kinase and PI3K [46]. Together, these findings show that resveratrol can induce autophagy and may have an important role in tumor therapy. However, resveratrol is mainly associated with protective autophagy, and was identified in the present study because we studied autophagy genes that were influenced by herbal monomers.

Curcumin is a class of phenolic pigment extracted from turmeric in the genus Curcuma and is a natural phenolic antioxidant [51]. Turmeric roots contain a high content of curcumin. Numerous in vivo and in vitro experiments have shown that curcumin participates in the regulation of multiple cellular pathways, such as NF-kB, AKT, MAPK, p53, JAK/STAT and AMPK [52]. Related reports have demonstrated that curcumin can inhibit the growth of malignant glioma by inducing autophagy [53]. Curcumin-induced autophagy can also reduce the survival of cancer cells in hollow organs [54]. Li et al. [55] found that, in SKN cells, curcumin induced autophagy by activating the ERK1/2 pathway. In addition, treatment with an ERK1/2 inhibitor could reduce the ability of curcumin to induce autophagy and increase the ability of curcumin to induce apoptosis. Yamauchi et al. [56] reported that curcumin could increase LC3-II/ LC3-I expression, induce the formation of autophagosomes and reduce ATG5 RNA silencing. Han et al. [57] found that curcumin prevented oxidative stress-induced endothelial cell damage by inducing autophagy. Together, these findings confirm that curcumin has an important role in the induction of autophagy.

Quercetin is a flavonoid antioxidant that is widely present in plants. Wang et al. [58] experimentally confirmed that quercetin could induce gastric cancer cells to form autophagic vacuoles, transform LC3-I into LC3II, and activate autophagy genes. In addition, Kim et al. [59] found that quercetin could induce autophagy in
U373MG cells, further confirming that quercetin initiates the autophagy process in gastric cancer cells.

Melatonin is an indole compound that has important physiological and pathological functions including regulating the body's circadian rhythms and seasonal rhythms. Choi et al. [60] found that in wild-type (WT) and GCD2homozygous $(\mathrm{HO})$ corneal fibroblast cell lines, melatonin could activate the initiation of autophagy. Recently, it was also reported that melatonin could prevent ischemiareperfusion injury of the brain through the induction of autophagy [61]. Chen et al. [62] further demonstrated that melatonin could increase the LC3-II to LC3-I ratio and enhance autophagy. Overall, these findings show that melatonin plays an important role in the activation and initiation of autophagy.

In addition to the drugs mentioned above and shown to regulate autophagy, colchicine, patulin, chloramphenicol, genistein, L-arginine, benzo- $(\alpha)$-pyrene and others have been reported to induce apoptosis and may therefore demonstrate regulatory potential for autophagy. However, the exact effects of all these monomers on autophagy and on the crosstalk between autophagy and apoptosis need to be confirmed in vitro and in vivo. In addition, autophagy may either promote cell survival or cell death, and the delicate balance between the two is ill understood [8]. The present study revealed possible effects of some monomers on genes involved in autophagy, but the results do not allow determining if these monomers would tip the balance one way or the other. Future studies are necessary to address these issues.

Colchicine is an alkaloid extracted from the plant Colchicum autumnale in the lily family [63]. Chen et al. [64] showed that colchicine could induce apoptosis through the mitochondrial pathway in L-02 cells. Patulin is a class of mycotoxin found in apples, grapes, pears and peaches, andt is an effective genotoxic compound. Saxena et al. [65] reported that patulin could induce DNA damage, resulting in p53-mediated cell cycle arrest and subsequent apoptosis. Kwon et al. [66] demonstrated that patulin acts via oxidative stress to induce phosphorylation of the transcription factor EGR-1 and thus increases the expression of ATF3, leading to cell cycle arrest and activation of the apoptotic protein cascade. Taurine, also known as $\beta$-aminoethanesulfonic acid, was first isolated from calculus bovis. Taurine is chemically stable and is a sulfur-containing non-protein amino acid that is closely associated with human metabolism. Zhang et al. [67] demonstrated that taurine could induce apoptosis in pulmonary artery smooth muscle cells via the death-receptor pathway. L-arginine is a semi-essential amino acid that has nutritional and immune regulation functions, can act as a precursor for the synthesis of nitric oxide, and is involved in various physiological and pathological processes in the body. Shu [68] found that L-arginine induces 
apoptosis by downregulating the expression and survival of the anti-apoptotic protein $\mathrm{Bcl}-2$ and upregulating the expression of the pro-apoptotic protein p53. Genistein is an isoflavone found in soy and is a weak plant estrogen. Recent studies from Xia et al. [69] found that genistein could increase the expression of miR-34a, thereby inhibiting the expression of Notch-1 and promoting apoptosis. Kayisli et al. [70] demonstrated experimentally that genistein acts as an anti-proliferative factor and can stimulate human coronary artery endothelial cell apoptosis.

Therefore, some of the above-described herbal monomers deduced from the disease-gene-drug network have been shown to regulate the occurrence of autophagy, thus confirming the validity of this method. Some other herbal monomers deduced in this study have been used in the study of apoptosis but have not been reported to function in the regulation of autophagy, suggesting that we may find new drugs that can regulate autophagy among these herbal monomers.

The present study suffers from some limitations. Indeed, the aim of the present study was to use a new approach to screen for herbal monomers that could influence autophagy genes. However, each of these monomers will have to be carefully studied to determine their exact effects in vitro and in vivo and which way they normally tip autophagy (cell survival or death), and if the balance could be tipped the other way in specific circumstances. In addition, there is crosstalk between autophagy and apoptosis $[9,10]$, and future studies should evaluate the effects of different compounds on this crosstalk.

\section{Conclusion}

This study provides a new strategy to discover herbal compounds for tumor treatment and disease alleviation that may assist with the identification of tumor cell autophagy inducers in the future.

\section{Competing interests}

The authors declare that they have no competing interests.

\section{Authors' contributions}

$\mathrm{WHZ}$ and DBX planned, designed and conceived the study. CJH and ZPY drafted the manuscript. BG and XZM contributed to the interpretation of the data and revised the manuscript. ML and $X Q$ performed bioinformatics analyses. All authors read and approved the final manuscript.

\section{Authors' information \\ Chenjun Hao is a medical doctor candidate, Zhengpeng Yang is a medical master candidate, Bo Gao is a medical doctor candidate, Xianzhi Meng is an associate professor, Dongbo Xue is a professor, and Weihui Zhang is a professor. The above authors are all from the Department of General Surgery, the First Affiliated Hospital of Harbin Medical University, Harbin, China. \\ Ming Lu is a assistant research fellow, Xin Qiao is a assistant research fellow, they both from Department of Surgery, David Geffen School of Medicine, University of California at Los Angeles, Los Angeles, CA, USA.}

\section{Acknowledgements}

This work was supported by the National Natural Science Foundation of China (81370566, 81170397).

\section{Author details}

'Department of General Surgery, the First Affiliated Hospital of Harbin Medical University, Harbin, China. ${ }^{2}$ Department of Surgery, David Geffen School of Medicine, University of California at Los Angeles, Los Angeles, CA, USA.

Received: 6 July 2014 Accepted: 27 November 2014

Published: 4 December 2014

\section{References}

1. Fitzpatrick FA, Wheeler R: The immunopharmacology of paclitaxel (Taxol), docetaxel (Taxotere), and related agents. Int Immunopharmacol 2003, 3:1699-1714.

2. Rates SM: Plants as source of drugs. Toxicon 2001, 39:603-613.

3. Degenhardt K, Mathew R, Beaudoin B, Bray K, Anderson D, Chen G, Mukherjee C, Shi Y, Gélinas C, Fan Y, Nelson DA, Jin S, White E: Autophagy promotes tumor cell survival and restricts necrosis, inflammation, and tumorigenesis. Cancer Cell 2006, 10:51-64

4. Qu X, Yu J, Bhagat G, Furuya N, Hibshoosh H, Troxel A, Rosen J, Eskelinen EL, Mizushima N, Ohsumi Y, Cattoretti G, Levine B: Promotion of tumorigenesis by heterozygous disruption of the beclin 1 autophagy gene. J Clin Invest 2003, 112:1809-1820.

5. Tsujimoto Y, Shimizu S: Another way to die: autophagic programmed cell death. Cell Death Differ 2005, 12(Suppl 2):1528-1534.

6. Ryter SW, Mizumura K, Choi AM: The impact of autophagy on cell death modalities. Int J Cell Biol 2014, 2014:502676.

7. Altman BJ, Rathmell JC: Metabolic stress in autophagy and cell death pathways. Cold Spring Harb Perspect Biol 2012, 4:a008763.

8. Klionsky DJ, Abdalla FC, Abeliovich H, Abraham RT, Acevedo-Arozena A, Adeli K, Agholme L, Agnello M, Agostinis P, Aguirre-Ghiso JA, Ahn HJ, Ait-Mohamed O, Ait-Si-Ali S, Akematsu T, Akira S, Al-Younes HM, Al-Zeer MA, Albert ML, Albin RL, Alegre-Abarrategui J, Aleo MF, Alirezaei M, Almasan A, Almonte-Becerril M, Amano A, Amaravadi R, Amarnath S, Amer AO, Andrieu-Abadie N, Anantharam V, et al: Guidelines for the use and interpretation of assays for monitoring autophagy. Autophagy 2012, 8:445-544.

9. Su M, Mei Y, Sinha S: Role of the crosstalk between autophagy and apoptosis in cancer. J Oncol 2013, 2013:102735.

10. Gordy C, He YW: The crosstalk between autophagy and apoptosis: where does this lead? Protein Cell 2012, 3:17-27.

11. Dengjel J, Schoor O, Fischer R, Reich M, Kraus M, Müller M, Kreymborg K, Altenberend F, Brandenburg J, Kalbacher H, Brock R, Driessen C, Rammensee HG, Stevanovic S: Autophagy promotes MHC class II presentation of peptides from intracellular source proteins. Proc Natl Acad Sci U S A 2005, 102:7922-7927.

12. Matarrese P, Tinari A, Ascione B, Gambardella L, Remondini D, Salvioli S, Tenedini E, Tagliafico E, Franceschi C, Malorni W: Survival features of EBV-stabilized cells from centenarians: morpho-functional and transcriptomic analyses. Age (Dordr) 2012, 34:1341-1359.

13. Keshava Prasad TS, Goel R, Kandasamy K, Keerthikumar S, Kumar S, Mathivanan S, Telikicherla D, Raju R, Shafreen B, Venugopal A, Balakrishnan L, Marimuthu A, Banerjee S, Somanathan DS, Sebastian A, Rani S, Ray S, Harrys Kishore CJ, Kanth S, Ahmed M, Kashyap MK, Mohmood R, Ramachandra YL, Krishna V, Rahiman BA, Mohan S, Ranganathan P, Ramabadran S, Chaerkady R, Pandey A: Human protein reference database-2009 update. Nucleic Acids Res 2009, 37:D767-772.

14. Adamcsek B, Palla G, Farkas IJ, Derenyi I, Vicsek T: CFinder: locating cliques and overlapping modules in biological networks. Bioinformatics 2006, 22:1021-1023.

15. Smoot ME, Ono K, Ruscheinski J, Wang PL, Ideker T: Cytoscape 2.8: new features for data integration and network visualization. Bioinformatics 2011, 27:431-432.

16. Yang S, Kimmelman AC: A critical role for autophagy in pancreatic cancer. Autophagy 2011, 7:912-913.

17. Toth S, Nagy K, Palfia Z, Rez G: Cellular autophagic capacity changes during azaserine-induced tumour progression in the rat pancreas. Up-regulation in all premalignant stages and down-regulation with loss of cycloheximide sensitivity of segregation along with malignant transformation. Cell Tissue Res 2002, 309:409-416.

18. Hait WN, Jin S, Yang JM: A matter of life or death (or both): understanding autophagy in cancer. Clin Cancer Res 2006, 12:1961-1965. 
19. Bursch W, Ellinger A, Kienzl H, Torok L, Pandey S, Sikorska M, Walker R, Hermann RS: Active cell death induced by the anti-estrogens tamoxifen and ICI 164384 in human mammary carcinoma cells (MCF-7) in culture: the role of autophagy. Carcinogenesis 1996, 17:1595-1607.

20. Qian W, Liu J, Jin J, Ni W, Xu W: Arsenic trioxide induces not only apoptosis but also autophagic cell death in leukemia cell lines via up-regulation of Beclin-1. Leuk Res 2007, 31:329-339.

21. Kim KW, Mutter RW, Cao C, Albert JM, Freeman M, Hallahan DE, Lu B: Autophagy for cancer therapy through inhibition of pro-apoptotic proteins and mammalian target of rapamycin signaling. J Biol Chem 2006, 281:36883-36890

22. Liu X, Yu H, Yang W, Zhou X, Lu H, Shi D: Mutations of NFKBIA in biopsy specimens from Hodgkin lymphoma. Cancer Genet Cytogenet 2010, 197:152-157.

23. Yang $S$, Bing $M$, Chen $F$, Sun $Y$, Chen $H$, Chen $W$ : Autophagy regulation by the nuclear factor kappaB signal axis in acute pancreatitis. Pancreas 2012, 41:367-373.

24. Gao J, Pfeifer D, He LJ, Qiao F, Zhang Z, Arbman G, Wang ZL, Jia CR, Carstensen J, Sun XF: Association of NFKBIA polymorphism with colorectal cancer risk and prognosis in Swedish and Chinese populations. Scand J Gastroenterol 2007, 42:345-350.

25. Rinkenbaugh AL, Baldwin AS: Monoallelic deletion of NFKBIA in glioblastoma: when less is more. Cancer Cell 2011, 19:163-165.

26. Tamura RE, de Vasconcellos JF, Sarkar D, Libermann TA, Fisher PB, Zerbin LF: GADD45 proteins: central players in tumorigenesis. Curr Mol Med 2012, 12:634-651.

27. Corcelle E, Djerbi N, Mari M, Nebout M, Fiorini C, Fénichel P, Hofman P, Poujeol P, Mograbi B: Control of the autophagy maturation step by the MAPK ERK and p38: lessons from environmental carcinogens. Autophagy 2007, 3:57-59.

28. Zhang Y, Wu Y, Cheng Y, Zhao Z, Tashiro S, Onodera S, Ikejima T: Fas-mediated autophagy requires JNK activation in HeLa cells. Biochem Biophys Res Commun 2008, 377:1205-1210.

29. Gartel AL, Tyner AL: The role of the cyclin-dependent kinase inhibitor p21 in apoptosis. Mol Cancer Ther 2002, 1:639-649.

30. Fujiwara K, Daido S, Yamamoto A, Kobayashi R, Yokoyama T, Aoki H, Iwado E, Shinojima N, Kondo Y, Kondo S: Pivotal role of the cyclin-dependent kinase inhibitor p21WAF1/CIP1 in apoptosis and autophagy. J Biol Chem 2008, 283:388-397.

31. Dunlop MG, Dobbins SE, Farrington SM, Jones AM, Palles C, Whiffin N, Tenesa A, Spain S, Broderick P, Ooi LY, Domingo E, Smillie C, Henrion M, Frampton M, Martin L, Grimes G, Gorman M, Semple C, Ma YP, Barclay E, Prendergast J, Cazier JB, Olver B, Penegar S, Lubbe S, Chander I, CarvajalCarmona LG, Ballereau S, Lloyd A, Vijayakrishnan J, et al: Common variation near CDKN1A, POLD3 and SHROOM2 influences colorectal cancer risk. Nat Genet 2012, 44:770-776.

32. Kibel AS, Suarez BK, Belani J, Oh J, Webster R, Brophy-Ebbers M, Guo C, Catalona WJ, Picus J, Goodfellow PJ: CDKN1A and CDKN1B polymorphisms and risk of advanced prostate carcinoma. Cancer Res 2003, 63:2033-2036.

33. Chimento SM, Kirsner RS: Understanding the role of c-Jun and Jun B transcription factors in skin cancer developments. J Invest Dermatol 2011 , 131:1002.

34. Langer $S$, Singer CF, Hudelist G, Dampier B, Kaserer $K$, Vinatzer $U$ Pehamberger $\mathrm{H}$, Zielinski C, Kubista E, Schreibner M: Jun and Fos family protein expression in human breast cancer: correlation of protein expression and clinicopathological parameters. Eur J Gynaecol Oncol 2006, 27:345-352.

35. Guo Y, Chang C, Huang R, Liu B, Bao L, Liu W: AP1 is essential for generation of autophagosomes from the trans-Golgi network. J Cell SCi 2012, 125:1706-1715.

36. Kojima E, Takeuchi A, Haneda M, Yagi A, Hasegawa T, Yamaki K, Takeda K, Akira S, Shimokata K, Isobe K: The function of GADD34 is a recovery from a shutoff of protein synthesis induced by ER stress: elucidation by GADD34-deficient mice. FASEB J 2003, 17:1573-1575.

37. Talloczy Z, Jiang W, Virgin HWT, Leib DA, Scheuner D, Kaufman RJ, Eskelinen EL, Levine B: Regulation of starvation- and virus-induced autophagy by the elF2alpha kinase signaling pathway. Proc Natl Acad Sci U S A 2002, 99:190-195.

38. Liu Y, Laszlo C, Liu Y, Liu W, Chen X, Evans SC, Wu S: Regulation of G(1) arrest and apoptosis in hypoxia by PERK and GCN2-mediated elF2alpha phosphorylation. Neoplasia 2010, 12:61-68.
39. Filippi-Chiela EC, Thome MP, Silva MM B e, Pelegrini AL, Ledur PF, Garicochea B, Zamin LL, Lenz G: Resveratrol abrogates the temozolomide-induced G2 arrest leading to mitotic catastrophe and reinforces the temozolomideinduced senescence in glioma cells. BMC Cancer 2013, 13:147.

40. Lin HY, Tang HY, Keating T, Wu YH, Shih A, Hammond D, Sun M, Hercbergs A, Davis FB, Davis PJ: Resveratrol is pro-apoptotic and thyroid hormone is anti-apoptotic in glioma cells: both actions are integrin and ERK mediated. Carcinogenesis 2008, 29:62-69.

41. Yamamoto M, Suzuki SO, Himeno M: Resveratrol-induced autophagy in human U373 glioma cells. Oncol Lett 2010, 1:489-493.

42. Choi MS, Kim Y, Jung JY, Yang SH, Lee TR, Shin DW: Resveratrol induces autophagy through death-associated protein kinase 1 (DAPK1) in human dermal fibroblasts under normal culture conditions. Exp Dermato/ 2013, 22:491-494.

43. Delmas D, Solary E, Latruffe N: Resveratrol, a phytochemical inducer of multiple cell death pathways: apoptosis, autophagy and mitotic catastrophe. Curr Med Chem 2011, 18:1100-1121.

44. Opipari AW Jr, Tan L, Boitano AE, Sorenson DR, Aurora A, Liu JR: Resveratrol-induced autophagocytosis in ovarian cancer cells. Cancer Res 2004, 64:696-703.

45. Kueck A, Opipari AW Jr, Griffith KA, Tan L, Choi M, Huang J, Wahl H, Liu JR: Resveratrol inhibits glucose metabolism in human ovarian cancer cells. Gynecol Oncol 2007, 107:450-457.

46. Trincheri NF, Follo C, Nicotra G, Peracchio C, Castino R, Isidoro C Resveratrol-induced apoptosis depends on the lipid kinase activity of Vps34 and on the formation of autophagolysosomes. Carcinogenesis 2008, 29:381-389.

47. Signorelli P, Munoz-Olaya JM, Gagliostro V, Casas J, Ghidoni R, Fabriàs G: Dihydroceramide intracellular increase in response to resveratrol treatment mediates autophagy in gastric cancer cells. Cancer Lett 2009, 282:238-243.

48. Ohshiro K, Rayala SK, El-Naggar AK, Kumar R: Delivery of cytoplasmic proteins to autophagosomes. Autophagy 2008, 4:104-106.

49. Ohshiro K, Rayala SK, Kondo S, Gaur A, Vadlamudi RK, El-Naggar AK, Kumar R: Identifying the estrogen receptor coactivator PELP1 in autophagosomes. Cancer Res 2007, 67:8164-8171.

50. Puissant A, Robert G, Fenouille N, Luciano F, Cassuto JP, Raynaud S, Auberger $P$ : Resveratrol promotes autophagic cell death in chronic myelogenous leukemia cells via JNK-mediated p62/SQSTM1 expression and AMPK activation. Cancer Res 2010, 70:1042-1052.

51. Palipoch S, Punsawad C, Koomhin P, Suwannalert P: Hepatoprotective effect of curcumin and alpha-tocopherol against cisplatin-induced oxidative stress. BMC Complement Altern Med 2014, 14:111.

52. El-Bahr SM: Curcumin regulates gene expression of insulin like growth factor, B-cell CLL/lymphoma 2 and antioxidant enzymes in streptozotocin induced diabetic rats. BMC Complement Altern Med 2013, 13:368.

53. Aoki H, Takada Y, Kondo S, Sawaya R, Aggarwal BB, Kondo Y: Evidence that curcumin suppresses the growth of malignant gliomas in vitro and in vivo through induction of autophagy: role of Akt and extracellular signal-regulated kinase signaling pathways. Mol Pharmacol 2007, 72:29-39.

54. Kim JY, Cho TJ, Woo BH, Choi KU, Lee CH, Ryu MH, Park HR: Curcumininduced autophagy contributes to the decreased survival of oral cancer cells. Arch Oral Biol 2012, 57:1018-1025.

55. Li B, Takeda T, Tsuiji K, Wong TF, Tadakawa M, Kondo A, Nagase S, Yaegashi $\mathrm{N}$ : Curcumin induces cross-regulation between autophagy and apoptosis in uterine leiomyosarcoma cells. Int J Gynecol Cancer 2013, 23:803-808.

56. Yamauchi $Y$, Izumi $Y$, Asakura K, Hayashi $Y$, Nomori H: Curcumin induces autophagy in ACC-MESO-1 cells. Phytother Res 2012, 26:1779-1783.

57. Han J, Pan XY, Xu Y, Xiao Y, An Y, Tie L, Pan Y, Li XJ: Curcumin induces autophagy to protect vascular endothelial cell survival from oxidative stress damage. Autophagy 2012, 8:812-825.

58. Wang K, Liu R, Li J, Mao J, Lei Y, Wu J, Zeng J, Zhang T, Wu H, Chen L, Huang $C$, Wei $Y$ : Quercetin induces protective autophagy in gastric cancer cells: involvement of Akt-mTOR- and hypoxia-induced factor 1alpha-mediated signaling. Autophagy 2011, 7:966-978.

59. Kim H, Moon JY, Ahn KS, Cho SK: Quercetin induces mitochondrial mediated apoptosis and protective autophagy in human glioblastoma U373MG cells. Oxid Med Cell Longev 2013, 2013:596496.

60. Choi SI, Kim KS, Oh JY, Jin JY, Lee GH, Kim EK: Melatonin induces autophagy via an mTOR-dependent pathway and enhances clearance of mutant-TGFBIp. J Pineal Res 2013, 54:361-372. 
61. Guo Y, Wang J, Wang Z, Yang Y, Wang X, Duan Q: Melatonin protects N2a against ischemia/reperfusion injury through autophagy enhancement. J Huazhong Univ Sci Technolog Med Sci 2010, 30:1-7.

62. Chen J, Wang L, Wu C, Hu Q, Gu C, Yan F, Li J, Yan W, Chen G: Melatoninenhanced autophagy protects against neural apoptosis via a mitochondrial pathway in early brain injury following a subarachnoid hemorrhage. J Pineal Res 2014, 56:12-19.

63. Niel E, Scherrmann JM: Colchicine today. Joint Bone Spine 2006, 73:672-678.

64. Chen XM, Liu J, Wang T, Shang J: Colchicine-induced apoptosis in human normal liver L-02 cells by mitochondrial mediated pathways. Toxicol In Vitro 2012, 26:649-655.

65. Saxena N, Ansari KM, Kumar R, Dhawan A, Dwivedi PD, Das M: Patulin causes DNA damage leading to cell cycle arrest and apoptosis through modulation of Bax, $\mathrm{p}(53)$ and $\mathrm{p}(21 / \mathrm{WAF} 1)$ proteins in skin of mice. Toxicol Appl Pharmacol 2009, 234:192-201.

66. Kwon O, Soung NK, Thimmegowda NR, Jeong SJ, Jang JH, Moon DO, Chung JK, Lee KS, Kwon YT, Erikson RL, Ahn JS, Kim BY: Patulin induces colorectal cancer cells apoptosis through EGR-1 dependent ATF3 up-regulation. Cell Signal 2012, 24:943-950.

67. Zhang X, Sheng J, Zhang C, Zhao F: Taurine induces apoptosis in pulmonary artery smooth muscle cells. Zhongguo Zhong Yao Za Zhi 2012, 37:654-657.

68. Shu XL, Xu H, Yu TT, Zhong JX, Lei T: Regulation of apoptosis in human gastric cancer cell line SGC-7901 by L-arginine. Panminerva Med 2014, 56:227-231

69. Xia J, Duan Q, Ahmad A, Bao B, Banerjee S, Shi Y, Ma J, Geng J, Chen Z, Rahman KM, Miele L, Sarkar FH, Wang Z: Genistein inhibits cell growth and induces apoptosis through up-regulation of miR-34a in pancreatic cancer cells. Curr Drug Targets 2012, 13:1750-1756.

70. Kayisli UA, Guzeloglu-Kayisli O, Guzel E, Arici A: Genistein inhibits cell proliferation and stimulates apoptosis in human coronary artery endothelial cells. Gynecol Obstet Invest 2013, 75:235-242.

doi:10.1186/1472-6882-14-466

Cite this article as: Hao et al:: Database screening of herbal monomers regulating autophagy by constructing a "disease-gene-drug" network. BMC Complementary and Alternative Medicine 2014 14:466.

\section{Submit your next manuscript to BioMed Central and take full advantage of:}

- Convenient online submission

- Thorough peer review

- No space constraints or color figure charges

- Immediate publication on acceptance

- Inclusion in PubMed, CAS, Scopus and Google Scholar

- Research which is freely available for redistribution 